\title{
Mucosal intralymphatic spread in a relapsed diffuse large B cell lymphoma
}

\author{
Shan-Chi Yu ${ }^{1,2,3} \cdot$ Chang-Tsu Yuan ${ }^{2,4,5}$ \\ Received: 16 April 2020 / Accepted: 5 June 2020 / Published online: 12 June 2020 \\ (C) Springer-Verlag GmbH Germany, part of Springer Nature 2020
}

\begin{abstract}
Intralymphatic spread is a rare finding and is associated with poor prognosis in diffuse large B cell lymphoma (DLBCL). Here, we report a case of relapsed DLBCL with mucosal intralymphatic spread. A 69-year-old man had been diagnosed with gastric DLBCL stage IIE at 57 years. He had a relapse with generalized lymphadenopathy and extranodal involvement at 61 years; then, second complete remission was achieved after salvage chemotherapy. He then had a second relapse with involvement of the terminal ileum, spinal cord, and left tonsil. The terminal ileum showed intralymphatic spread in the lamina propria of the intestinal villi, which was confirmed by D2-40 immunostaining. Eleven months later, another biopsy showed prominent intralymphatic spread in the mucosa of the terminal ileum. After salvage therapies, the spinal cord and tonsillar tumors resolved, but the intestinal tumors were refractory. The patient eventually died of progressive disease. In contrast to previously reported cases, the involved lymphatic vessels were observed in the mucosa, and the lymphoma cells expressed CD5 in the first colonoscopic biopsy. This rare case broadens the spectrum of intralymphatic spread in DLBCL.
\end{abstract}

Keywords Diffuse large B cell lymphoma · Endoscopic biopsy $\cdot$ Intralymphatic spread

\section{Introduction}

Diffuse large B cell lymphoma (DLBCL) is one of the most common histological types of lymphoma. The prognostic factors of DLBCL, not otherwise specified (NOS) are mostly clinical and molecular features. Immunohistochemically, CD5 positivity, MYC expression, and MYC and BCL2 coexpression are associated with poor prognosis in DLBCL [1-4]. Fluorescence in situ hybridization (FISH) has demonstrated that MYC

Shan-Chi Yu

b88401002@ntu.edu.tw

1 Department of Pathology and Graduate Institute of Pathology, College of Medicine, National Taiwan University, Taipei, Taiwan

2 Department of Pathology, National Taiwan University Hospital, No. Seven, Chung-Shan South Road, Taipei 10051, Taiwan

3 Graduate Institute of Microbiology, College of Medicine, National Taiwan University, Taipei, Taiwan

4 Department of Pathology, National Taiwan University Cancer Center, Taipei, Taiwan

5 Graduate Institute of Clinical Medicine, College of Medicine, National Taiwan University, Taipei, Taiwan rearrangement correlates with poor prognosis [5]. Regarding morphology, we previously observed that intralymphatic spread is a rare finding and is associated with poor prognosis in DLBCL [6]. In extranodal organs (for example, the gastrointestinal tract, breast, and female genital tract), we observed peritumoral lymphatic vessels were distended with lymphoma cells, reminiscent of tumor emboli of solid cancers [6]. In the previous case series, we found intralymphatic spread in various histological structures, but mucosa was generally spared. Correspondingly, the involved lymphatic vessels were observed exclusively in surgical resection specimens and not in any mucosal biopsy [6].

Here, we report a relapsed DLBCL with mucosal intralymphatic spread in serial colonoscopic biopsies. The morphology, immunophenotype, and clinical course differ from the cases of DLBCL with intralymphatic spread we reported previously [6].

\section{Clinical history}

69-year-old man with a history of DLBCL was admitted because of body weight loss and a positive fecal occult blood 
test. The patient was diagnosed with stage IIE gastric DLBCL at 57 years when he presented with a huge gastric tumor causing gastric outlet obstruction. He achieved the first complete remission after Helicobacter eradication therapy and chemotherapy. At 61 years, he had a relapse manifesting with generalized lymphadenopathy and involvement of the bone marrow, spleen, tonsils, colon, and lungs. After salvage chemotherapy, he remained in a second complete remission for 8 years. Colonoscopy revealed a $3-\mathrm{cm}$ ulcerative mass at the terminal ileum (Fig. 1a, b). Biopsies were taken at the ulcer margin.

A subsequent positron emission tomography scan revealed relapses at the terminal ileum, left tonsil, and spinal cord. He received salvage treatments including chemotherapy, radiotherapy, and polatuzumab vedotin. The tonsillar and spinal cord lesions resolved, but the intestinal tumor progressed. Eleven months after the first colonoscopic biopsy, the patient had tenesmus and abdominal pain. A computed tomography scan revealed marked wall thickening of the ileocecal region, right-sided colon, and rectum (Fig. 1c, d); no lymphadenopathy or hepatosplenomegaly was noted. He underwent another colonoscopy, which showed a 2-cm ulcer at the rectum and multiple polyps at the terminal ileum (Fig. 1e, f). Two months later, the patient died of progressive disease.

\section{Materials and methods}

The specimens were processed in the Department of Pathology, National Taiwan University Hospital. The
Fig. 1 The first colonoscopy showing an ulcerative mass at the terminal ileum (a). Biopsies taken at the ulcer margin (b). The subsequent computed tomography scan reveals marked wall thickening at the ileocecal region $(\mathbf{c})$ and rectum $(\mathbf{d})$. The second colonoscopy showing multiple small polyps at the edematous and erythematous mucosa of the terminal ileum (e, f)

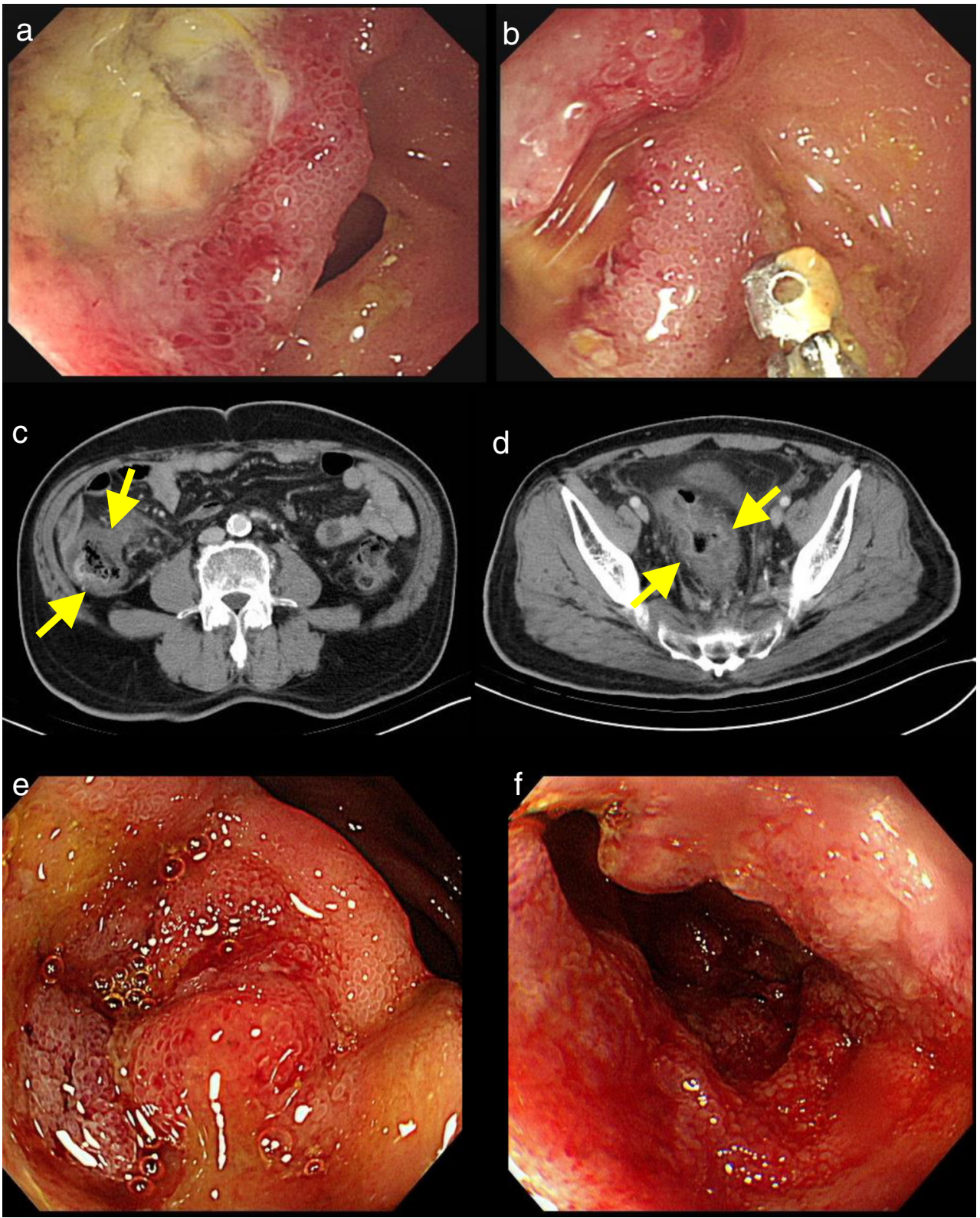


Fig. 2 The first biopsy of the terminal ileum showing DLBCL (a). Adjacent to the main tumor, intralymphatic spread is noted in the lamina propria of the intestinal villi $(\mathbf{b}, \mathbf{c}, \mathbf{d})$. The lymphoma cells are positive for $\mathrm{CD} 20$ (e) and CD5 (f), and negative for SOX11 (g). The lining cells of lymphatic vessels highlighted by D2-40 immunostaining (h)
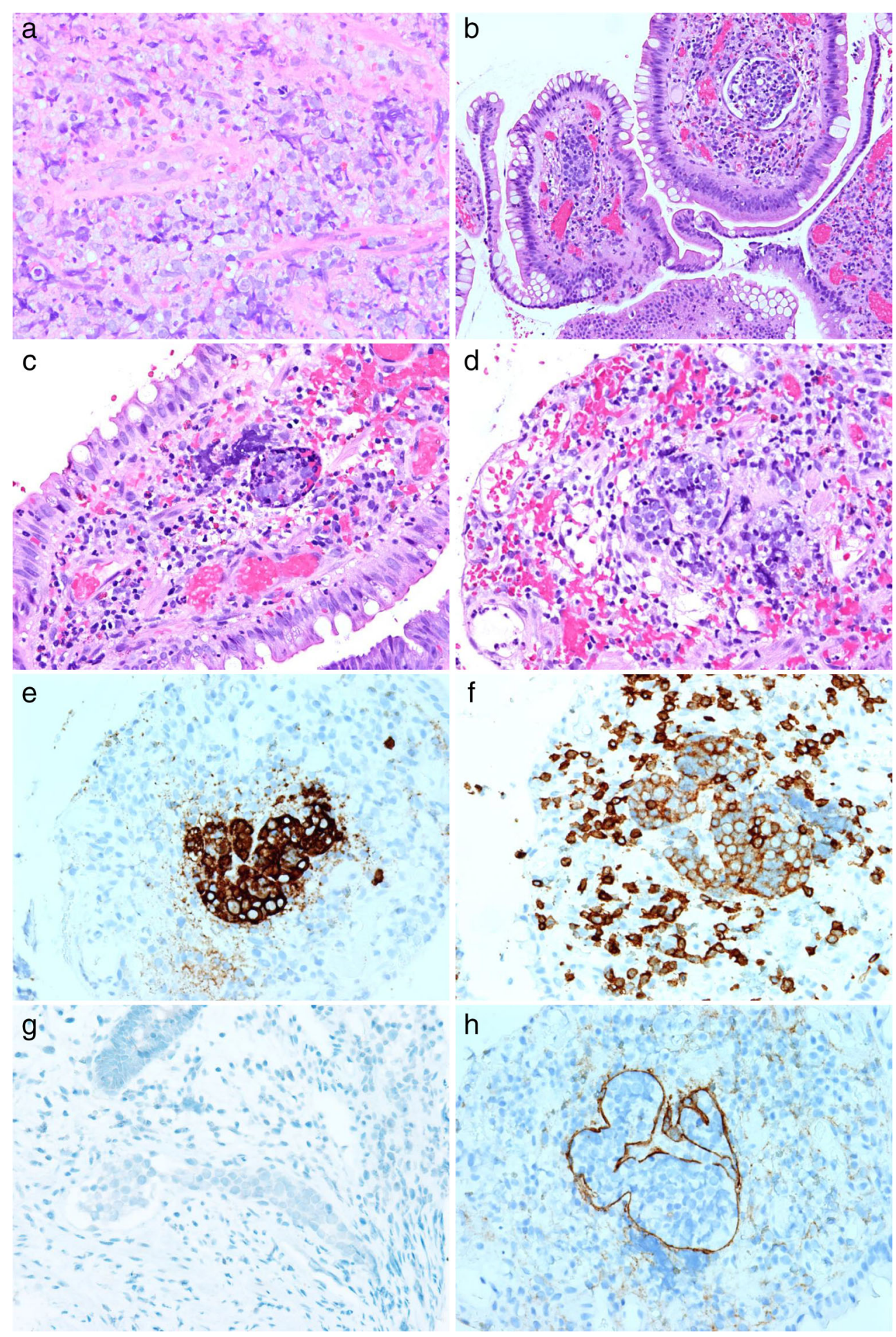

tissue sections were stained with hematoxylin and eosin and other special stains. The immunohistochemical stains and EBER in situ hybridization were performed on a Ventana autostainer (Roche, Tucson, AZ). FISH was performed for detecting MYC, BCL2, and BCL6 rearrangement.

\section{Results}

The first colonoscopic biopsy showed DLBCL, germinal center B cell-like by Hans algorithm [7]. Adjacent to the overt DLBCL, there were multiple well-circumscribed tiny nodules composed of atypical lymphoid cells in the intestinal villi (Fig. 2). The 
Fig. 3 The tongue base biopsy at the first relapse showing typical DLBCL (a). The lymphoma cells are CD5-negative (b)

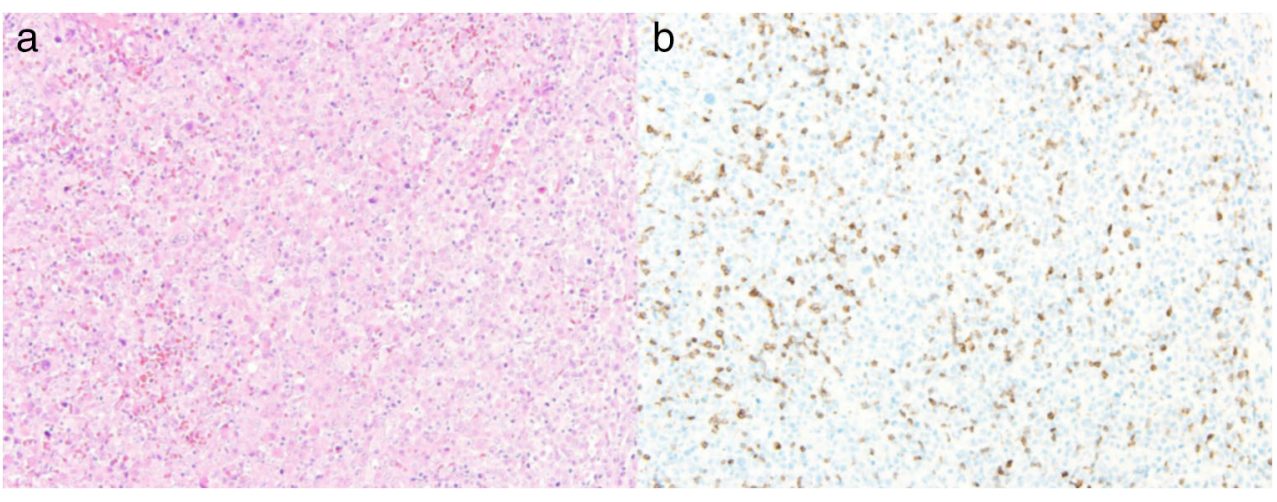

atypical lymphoid cells within the nodules displayed cytological features similar to those in the overt DLBCL. The nodules were surrounded by D2-40-positive cells, and the atypical lymphoid cells were positive for CD20, PAX5, CD10, BCL6, MUM1, and CD5 and negative for cyclin D1, SOX11, BCL2, c-myc, and EBER. FISH analysis revealed no rearrangement of MYC, BCL2, or BCL6. We think that these nodules were peritumoral lymphatic vessels distended with lymphoma cells, similar to intralymphatic spread that we reported previously [6]. Notably, the involved lymphatic vessels were located in the lamina propria of the intestinal mucosa, which we previously believed to be spared from intralymphatic spread.

The initial gastric biopsy was not available for review, as it had been performed in a private medical clinic. Reviewing the tonsillar and tongue base biopsies at the first relapse revealed a typical presentation of DLBCL (Fig. 3a). No intravascular growth or intralymphatic spread was noted. The lymphoma cells were CD5-negative (Fig. 3b), different from the first colonoscopic biopsy.

The second colonoscopic biopsy showed DLBCL at both the rectum and terminal ileum. Prominent intralymphatic spread was noted at the terminal ileum. In both the lamina propria and muscularis mucosa, numerous lymphatic vessels involved were present (Fig. $4 \mathrm{a}-\mathrm{c}$ ). Again, unlike the first colonoscopic biopsy, the lymphoma cells were CD5negative (Fig. 4d).

The disease course and microscopic findings are summarized in Fig. 5.

\section{Discussion}

The present case is relapsed DLBCL with peculiar morphology. The serial colonoscopic biopsies showed distended
Fig. 4 The second biopsy of the terminal ileum showing prominent intralymphatic spread (a). Lymphatic vessels lined by D2-40-positive endothelial cells (b). The lymphoma cells inside are positive for $\mathrm{CD} 20$ (c) and negative for CD5 (d)
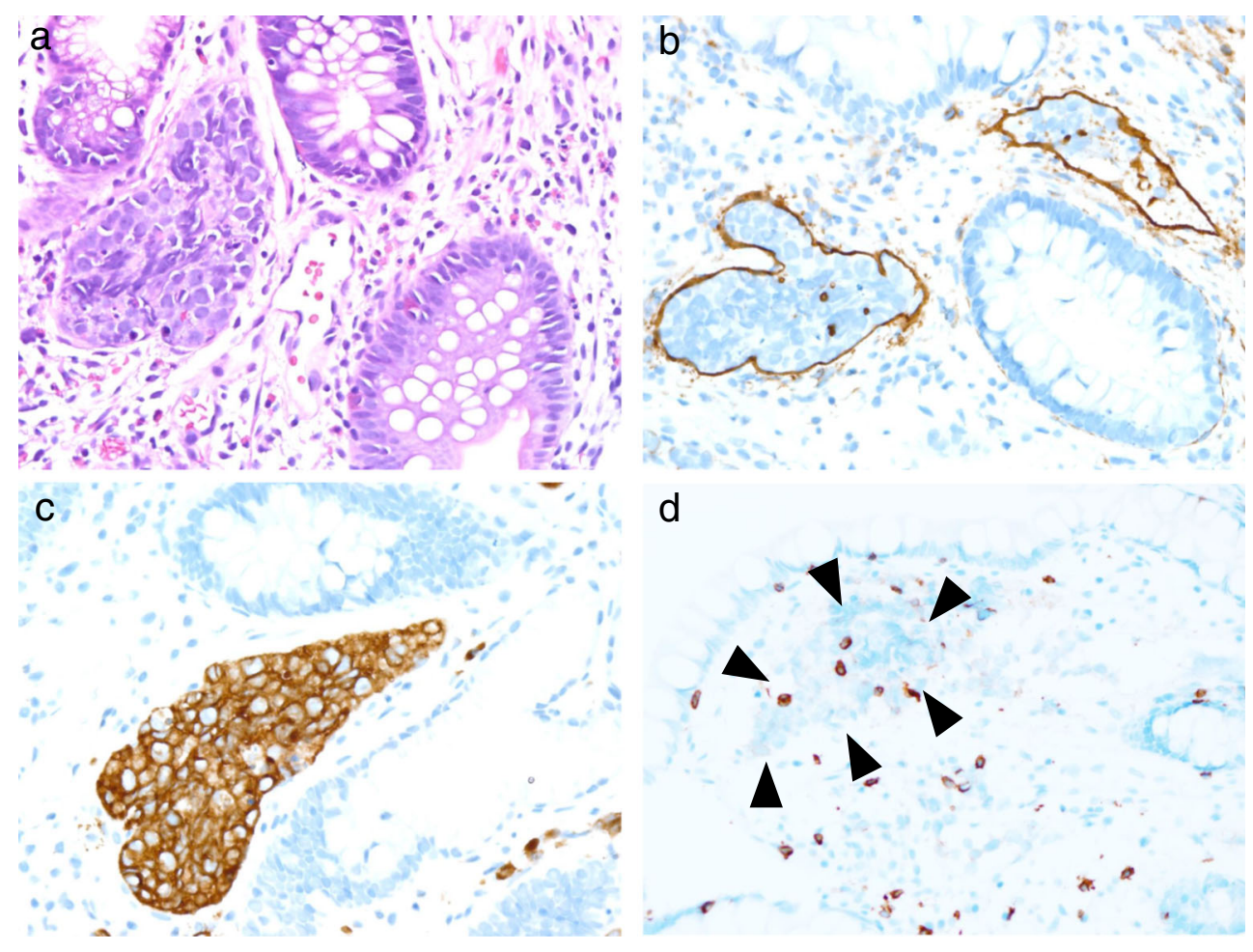
Fig. 5 Summary of the clinical course

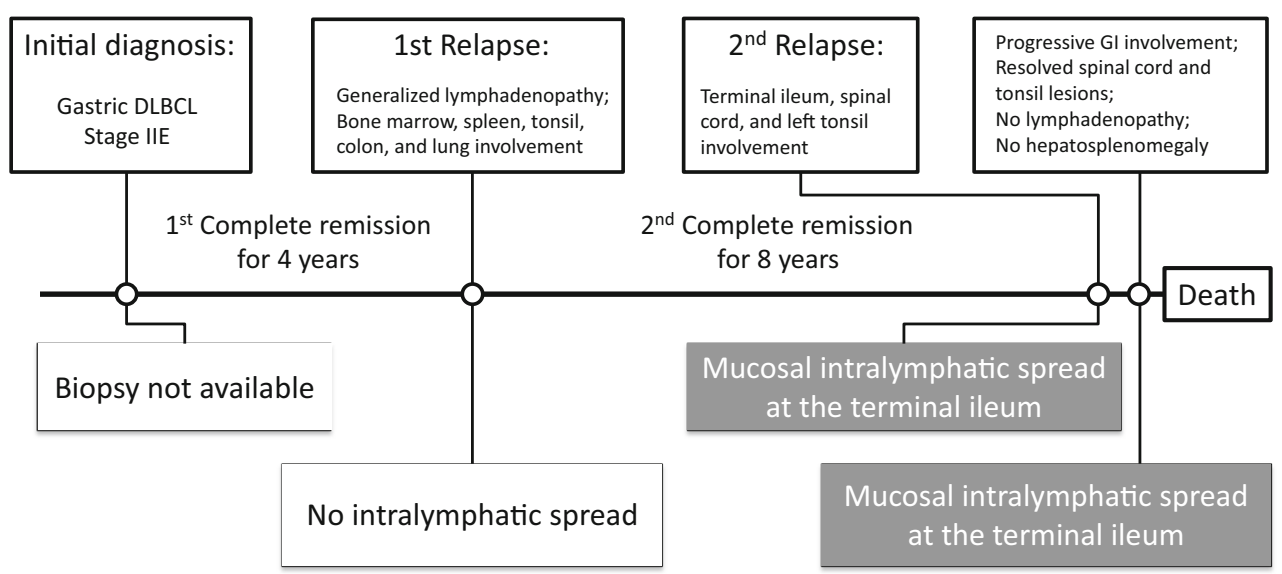

lymphatic vessels with lymphoma cells, similar to intralymphatic spread that we described previously [6]. However, the involved lymphatic vessels were located in the mucosa, mostly in the lamina propria and some in the muscularis mucosa. In the first terminal ileum biopsy, each intestinal villus contained a lymphoma nodule at the center, consistent with the histology of lacteal ducts. This rare growth pattern has never been reported for DLBCL.

The concept of intralymphatic spread in DLBCL is new; thus, DLBCL with intralymphatic spread may be confused with intravascular large B cell lymphoma (IVLBCL). However, their clinical and morphological features are very different. Clinically, IVLBCL presents with advanced stage disease in the skin, central nervous system, and lungs, with frequent bone marrow involvement and never lymphadenopathy, but DLBCL with intralymphatic spread tends to be localized at the gastrointestinal tract, female genital tract, and breast, some with regional lymphadenopathy but none with bone marrow involvement [6].

Morphologically, intralymphatic spread can usually be distinguished from intravascular growth through hematoxylin and eosin staining. In our opinion, the morphology of intralymphatic spread closely resembles lymphatic invasion of solid cancer. Intralymphatic spread typically presents as thin-walled vessels distended with lymphoma cells. Because lymphatic vessels generally parallel arteries and veins, uninvolved blood vessels can be discerned adjacent to the involved lymphatic vessels [6]. By contrast, the morphology of IVLBCL is more variable. Lymphoma cells may be located at the center of the vascular lumen, completely fill the lumen, or adhere to the endothelial surface [8]. The blood vessels are not always distended, which is very different from the morphology of intralymphatic spread. For ambiguous cases, D2-40 is the key immunohistochemical marker to distinguish lymphatics from blood vessels. Although CD5 is expressed in $38 \%$ of IVLBCL cases [9], we found all cases of DLBCL with intralymphatic spread were CD5-negative [6].
Although Ponzoni et al. stated that IVLBCL may be detected in the lamina propria of the gastrointestinal tract [10], very few cases of IVLBCL have been reported in the small intestine $[11,12]$. Based on limited microscopic photographs [12], we still found very different morphologies between IVLBCL and intralymphatic spread (Fig. 6). In the small intestinal mucosa, each villus contained a lacteal duct surrounded by a capillary network. Correspondingly, our case with intralymphatic spread presented with one involved lymphatic vessel in each intestinal villus, whereas in IVLBCL, multiple blood vessels are involved in each intestinal villus [12].

In our previous case series, the ileocecal region was the most common site of intralymphatic spread, but the mucosal lymphatic vessels were generally spared [6]. We speculate several explanations for mucosal sparing of intralymphatic spread. First, in our previous case series, many cases manifested with a large ulcerative tumor, where the overlying mucosa was largely necrotic. Second, normal mucosa is devoid of lymphatic vessels [13-15]; hence, lymphatic spread of DLBCL or lymphatic invasion by solid cancer is rarely observed in the mucosa. As an exception, the small intestinal mucosa is rich in lacteal ducts, which might explain our findings in this case. Third, unlike reticulate lymphatic networks in the submucosa and muscularis propria, lacteals are blindended ducts [16], whose structure might be less communicable to the main tumor. This is the first time we observed mucosal intralymphatic spread. Although the specimen size was small, the amount of involved lymphatic vessels was greater than that in many surgical resection specimens in our previous case series. We speculate this case might have extensive intralymphatic spread involving many layers of intestinal wall and not exclusively in the mucosa.

We previously found that all cases of DLBCL with intralymphatic spread were CD5-negative [6]. However, this unique case was $\mathrm{CD} 5$-positive in the first colonscopic biopsy. 
H\&E stain

Tangential section

Cross-section

a

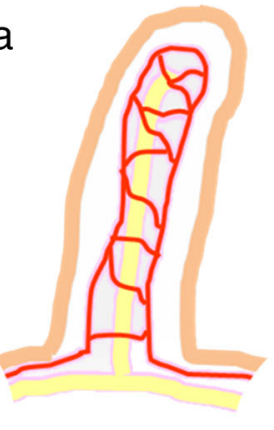

Epithelium

Lamina propria

\section{Blood \\ Lymph}

Lymphoma

Wall of blood vessels/ Lymphatic vessels

Fig. 6 Normal intestinal villus containing a lacteal duct surrounded by a capillary network (a). During intralymphatic spread, the lacteal duct is distended with lymphoma cells (b). In tissue sections, each villus contains an involved lymphatic vessel surrounded by many uninvolved blood

Approximately 5\% of DLBCL cases are CD5-positive, and CD5 expression is associated with poor prognosis in DLBCL, NOS [1]. Murase et al. have reported CD5 expression in 38\% of IVLBCL cases [9], which is a higher rate than conventional DLBCL. In the present case, the relationship between CD5 expression and the peculiar growth pattern are unclear.

We previously found intralymphatic spread in nine cases of de novo DLBCL and one case of large cell transformation of low-grade B cell lymphoma [6]. For de novo DLBCL receiving frontline rituximab-containing chemotherapy, intralymphatic spread was associated with poor prognosis [6]. The present case with mucosal intralymphatic spread was relapsed DLBCL that received second- and third-line therapies. Although the tonsillar and spinal cord lesions responded well to salvage therapy, the intestinal tumors were refractory. The different treatment responses suggest intratumoral heterogeneity. Because mucosal intralymphatic spread was observed exactly at the refractory part, the peculiar morphology could be associated with the poor treatment response observed in our patient.

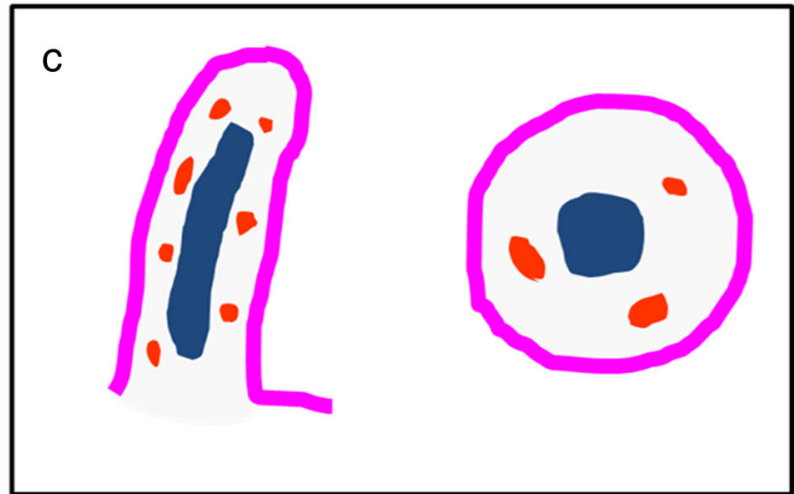

Epithelium Lymphoma Blood

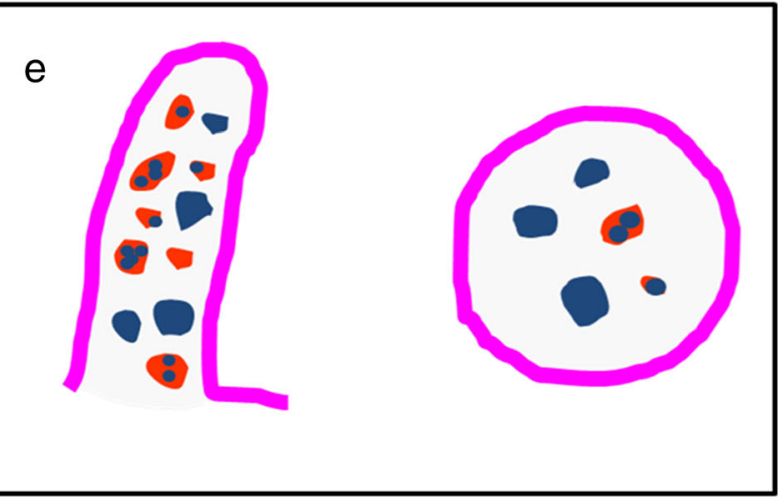

vessels (c). In IVLBCL, a variable amount of lymphoma cells are present in the capillaries (d). In tissue sections, each villus contains many involved blood vessels, some with only few lymphoma cells (e)

In addition to DLBCL, intralymphatic spread of lymphoma has been noted in primary cutaneous anaplastic large B cell lymphoma $[17,18]$. Based on morphology, we speculate that intralymphatic spread could be either spreading of lymphoma cells through lymphatic vessels or stasis of lymphoma cells in peritumoral lymphatic vessels. Mechanisms such as lymphangiogenesis and lymphocyte homing might be involved; hence, this finding could result from changes in the lymphoma cells themselves as well as remodeling of the microenvironment. The prognostic effect could be directly or indirectly related to these changes.

In summary, we report relapsed DLBCL with mucosal intralymphatic spread and CD5 expression. This rare case broadens the spectrum of extranodal intralymphatic spread in lymphomas/lymphoproliferative disorders.

\section{Compliance with ethical standards}

Conflict of interest The authors declare that they have no competing interests. 


\section{References}

1. Xu-Monette ZY, Tu M, Jabbar KJ, Cao X, Tzankov A, Visco C, Nagarajan L, Cai Q, Montes-Moreno S, An Y, Dybkaer K, Chiu A, Orazi A, Zu Y, Bhagat G, Richards KL, Hsi ED, Choi WW, van Krieken J, Huh J, Ponzoni M, Ferreri AJ, Zhao X, Møller MB, Farnen JP, Winter JN, Piris MA, Miranda RN, Medeiros LJ, Young KH (2015) Clinical and biological significance of de novo CD5+ diffuse large B-cell lymphoma in Western countries. Oncotarget 6:5615-5633

2. Xu-Monette ZY, Dabaja BS, Wang X, Tu M, Manyam GC, Tzankov A, Xia Y, Zhang L, Sun R, Visco C, Dybkaer K, Yin L, Chiu A, Orazi A, Zu Y, Bhagat G, Richards KL, Hsi ED, Choi WWL, van Krieken JH, Huh J, Ponzoni M, Ferreri AJM, Møller MB, Parsons BM, Zhao X, Winter JN, Piris MA, McDonnell TJ, Miranda RN, Li Y, Medeiros LJ, Young KH (2015) Clinical features, tumor biology, and prognosis associated with MYC rearrangement and Myc overexpression in diffuse large B-cell lymphoma patients treated with rituximab-CHOP. Mod Pathol 28:15551573

3. Johnson NA, Slack GW, Savage KJ, Connors JM, Ben-Neriah S, Rogic S, Scott DW, Tan KL, Steidl C, Sehn LH, Chan WC, Iqbal J, Meyer PN, Lenz G, Wright G, Rimsza LM, Valentino C, Brunhoeber P, Grogan TM, Braziel RM, Cook JR, Tubbs RR, Weisenburger DD, Campo E, Rosenwald A, Ott G, Delabie J, Holcroft C, Jaffe ES, Staudt LM, Gascoyne RD (2012) Concurrent expression of MYC and BCL2 in diffuse large B-cell lymphoma treated with rituximab plus cyclophosphamide, doxorubicin, vincristine, and prednisone. J Clin Oncol 30:3452-3459

4. Staiger AM, Ziepert M, Horn H, Scott DW, Barth TFE, Bernd HW, Feller AC, Klapper W, Szczepanowski M, Hummel M, Stein H, Lenze D, Hansmann ML, Hartmann S, Möller P, Cogliatti S, Lenz G, Trümper L, Löffler M, Schmitz N, Pfreundschuh M, Rosenwald A, Ott G, for the German High-Grade Lymphoma Study Group (2017) Clinical impact of the cell-of-origin classification and the MYC/ BCL2 dual expresser status in diffuse large B-cell lymphoma treated within prospective clinical trials of the German HighGrade Non-Hodgkin's Lymphoma Study Group. J Clin Oncol 35: $2515-2526$

5. Li S, Weiss VL, Wang XJ, Desai PA, Hu S, Yin CC, Tang G, Reddy NM, Medeiros LJ, Lin P (2016) High-grade B-cell lymphoma with MYC rearrangement and without BCL2 and BCL6 rearrangements is associated with high P53 expression and a poor prognosis. Am J Surg Pathol 40:253-261

6. Cheng CL, Su YC, Chao TY, Lin CW, Chou SC, Yao M, Kuo SH, $\mathrm{Yu}$ SC (2018) Intralymphatic spread is a rare finding associated with poor prognosis in diffuse large B-cell lymphoma with extranodal involvements. Am J Surg Pathol 42:616-624

7. Hans CP, Weisenburger DD, Greiner TC, Gascoyne RD, Delabie J, Ott G, Müller-Hermelink HK, Campo E, Braziel RM, Jaffe ES, Pan Z, Farinha P, Smith LM, Falini B, Banham AH, Rosenwald A, Staudt LM, Connors JM, Armitage JO, Chan WC (2004) Confirmation of the molecular classification of diffuse large Bcell lymphoma by immunohistochemistry using a tissue microarray. Blood 103:275-282

8. Ponzoni M, Campo E, Nakamura S (2018) Intravascular large Bcell lymphoma: a chameleon with multiple faces and many masks. Blood 132:1561-1567

9. Murase T, Yamaguchi M, Suzuki R, Okamoto M, Sato Y, Tamaru JI, Kojima M, Miura I, Mori N, Yoshino T, Nakamura S (2007) Intravascular large B-cell lymphoma (IVLBCL): a clinicopathologic study of 96 cases with special reference to the immunophenotypic heterogeneity of CD5. Blood 109:478-485

10. Ponzoni M, Ferreri AJ (2006) Intravascular lymphoma: a neoplasm of 'homeless' lymphocytes? Hematol Oncol 24:105-112

11. Shimoyama Y, Sugimoto K, Kotake M, Uehara D, Kamide Y, Kuribayashi S, Kawamura O, Kusano M, Handa H, Hirato J, Yamada M (2015) Two cases of intravascular lymphoma diagnosed by gastrointestinal endoscopic biopsy. Intern Med 54: 3145-3149

12. Linnik Y, Rand J, Kaur P, Liu X (2017) Intravascular large B cell lymphoma diagnosed in random duodenal biopsies. A case report and a literature review. Virchows Arch 471:429-431

13. Kenney BC, Jain D (2008) Identification of lymphatics within the colonic lamina propria in inflammation and neoplasia using the monoclonal antibody D2-40. Yale J Biol Med 81:103-113

14. Listrom MB, Fenoglio-Preiser CM (1987) Lymphatic distribution of the stomach in normal, inflammatory, hyperplastic, and neoplastic tissue. Gastroenterology 93:506-514

15. Tomita T, Mah K (2015) Lymphatic vessels in the human endometrium: are they present or absent? J Clin Exp Pathol 5:241-242

16. Miller MJ, McDole JR, Newberry RD (2010) Microanatomy of the intestinal lymphatic system. Ann N Y Acad Sci 1207(Suppl 1): E21-E28

17. Gratzinger D, Million L, Kim YH (2015) Occult dermal lymphatic involvement is frequent in primary cutaneous anaplastic large cell lymphoma. Am J Dermatopathol 37:767-770

18. Ferrara G, Ena L, Cota C, Cerroni L (2015) Intralymphatic spread is a common finding in cutaneous CD30+ lymphoproliferative disorders. Am J Surg Pathol 39:1511-1517

Publisher's note Springer Nature remains neutral with regard to jurisdictional claims in published maps and institutional affiliations. 